\title{
Urinary Metabolomics on the Biochemical Profiles in Diet-Induced Hyperlipidemia Rat Using Ultraperformance Liquid Chromatography Coupled with Quadrupole Time-of-Flight SYNAPT High-Definition Mass Spectrometry
}

\author{
Hua Miao, ${ }^{1}$ Hua Chen, ${ }^{2}$ Xu Zhang, ${ }^{1}$ Lu Yin, ${ }^{2}$ Dan-Qian Chen, ${ }^{2}$ \\ Xian-Long Cheng, ${ }^{3} \mathrm{Xu} \mathrm{Bai}^{4}$ and Feng Wei ${ }^{3}$ \\ ${ }^{1}$ Department of Physical Education, Northwest University, No. 229 Taibai North Road, Xian, Shaanxi 710069, China \\ ${ }^{2}$ Department of Traditional Chinese Medicine, The College of Life Sciences, Northwest University, \\ No. 229 Taibai North Road, Xian, Shaanxi 710069, China \\ ${ }^{3}$ National Institutes for Food and Drug Control, State Food and Drug Administration, 2 Tiantan Xili, \\ Beijing 100050, China \\ ${ }^{4}$ Solution Centre, Waters Technologies (Shanghai) Ltd., No. 1000 Jinhai Road, Shanghai 201203, China
}

Correspondence should be addressed to Feng Wei; weifengs@gmail.com

Received 8 January 2014; Accepted 23 January 2014; Published 18 March 2014

Academic Editor: Ying-Yong Zhao

Copyright (C) 2014 Hua Miao et al. This is an open access article distributed under the Creative Commons Attribution License, which permits unrestricted use, distribution, and reproduction in any medium, provided the original work is properly cited.

\begin{abstract}
Ultraperformance liquid chromatography coupled with quadrupole time-of-flight synapt high-definition mass spectrometry metabolomics was used to characterize the urinary metabolic profiling of diet-induced hyperlipidaemia in a rat model. Analysis was done by orthogonal partial least squares discriminant analysis, correlation analysis, heat map analysis, and KEGG pathways analysis. Potential biomarkers were chosen by S-plot and were identified by accurate mass, isotopic pattern, and MS/MS fragments information. Significant differences in fatty acid, amino acid, nucleoside, and bile acid were observed, indicating the perturbations of fatty acid, amino acid, nucleoside, and bile acid metabolisms in diet-induced hyperlipidaemia rats. This study provides further insight into the metabolic profiling across a wide range of biochemical pathways in response to diet-induced hyperlipidaemia.
\end{abstract}

\section{Introduction}

Metabolomics is the quantitative measurement of the dynamic multiparametric metabolic responses of living systems to pathophysiological stimuli or genetic modifications [1]. Metabolomics is based on the determination of global metabolite profiles in biological fluids and tissues with subsequent data analysis via a range of multivariate statistical approaches [2]. As a powerful analytical platform, the application of metabolomics has dramatically increased in the fields of physiological evaluation, disease diagnosis, disease prognosis, therapy, biomarker discovery, drug therapy monitoring, and safety and toxicity evaluation [3].
Hyperlipidaemia, as a major risk factor of coronary heart disease, is one of the most important public health problems, with increasing rates of incidence and prevalence [4]. Hyperlipidaemia is defined as a disorder of lipid metabolism leading to abnormal increase of triglycerides (TG), total cholesterol (TC), low-density lipoprotein cholesterol (LDLC), very low-density lipoprotein cholesterol (VLDL-C), and decrease of high-density lipoprotein cholesterol (HDL-C) [5] . As a progressive chronic and metabolic disease, cardiovascular disease begins in adult and progresses to morbidity and mortality throughout the lifespan. Hyperlipidaemia has an important effect on development and progression of various cardiovascular diseases and atherosclerosis. Both moderate 
hyperlipidaemia and severe hyperlipidaemia are associated with cardiovascular disease [6]. Recent study indicates that a fundamental defect is an overproduction of large VLDL-C, which triggers a sequence of lipoprotein changes, leading to increased remnant particles, smaller LDL-C, and decreased HDL-C [7]. LDL-C is the primary target for the lipidlowering therapy and cardiovascular diseases prevention.

Mass spectrometry (MS) and proton nuclear magnetic resonance $\left({ }^{1} \mathrm{H} \mathrm{NMR}\right)$ spectroscopy are two analytical tools commonly used in metabolomics. Recently, an increasing number of ${ }^{1} \mathrm{H}$ NMR and gas chromatography-MS (GC-MS) based on metabolomics have been conducted to characterize hyperlipidaemia models and to assess drug treatment [8-12].

Proteomic profiling from insulin resistance and metabolic dyslipidemia rats demonstrated hepatic ER proteins ERp29, ERp46, and ER60; TAP1 and glutamate dehydrogenase were downregulated, whereas P-glycoprotein, $\alpha$ glucosidase, protein disulfide isomerase, fibrinogen, GRP94, and apolipoprotein E were upregulated in the hepatic ER of the fructose-fed hamster [13]. ${ }^{1} \mathrm{H}$ NMR-based metabolomics indicated that major metabolic processes like Krebs cycle, cholesterol metabolism, and osmoregulation are perturbed in hypercholesterolaemic rats [9]. GC-MS based on metabolomics showed amino acids (alanine, valine, aspartic acid, phenylalanine, etc.), fatty acids (octadecadienoic acid, arachidonic acid, etc.), and propanoic acid, and glucose and cholesterol were identified as biomarkers in diet-induced hyperlipidaemia rats [12]. Among the analytical techniques in metabolomics research, liquid chromatography is recognized as one of the best analytical techniques in selectivity, sensitivity, and reproducibility [14]. Furthermore, among the various liquid chromatography platforms, ultraperformance liquid chromatography (UPLC) is considered to be suitable for metabolite profiling and metabolomics study, especially for large-scale untargeted metabolic profiling due to its enhanced reproducibility of retention time. UPLC operates with sub$2 \mu \mathrm{m}$ chromatographic particles and a fluid system capable of operating at pressures up to $15000 \mathrm{psi}$, providing an increased chromatographic resolution compared to conventional high performance liquid chromatography (HPLC) using larger particles.

In 2005, Wrona et al. introduced the mass spectrometry ${ }^{\text {ElevatedEnergy }}\left(\mathrm{MS}^{\mathrm{E}}\right)$ data collection technique [15], in which two scanning functions are simultaneously used for data collection. In other words, $\mathrm{MS}^{\mathrm{E}}$ can provide parallel alternating scans for acquisition at either low collision energy to obtain precursor ion information or high collision energy to obtain full-scan accurate mass fragment, precursor ion, and neutral loss information [14]. $\mathrm{MS}^{\mathrm{E}}$ involves a simultaneous acquisition through alternating between high and low collision energies during a single chromatographic run. This ability is of major importance, as it offers the structural information required for the identification of unknown biomarkers in the context of untargeted analyses. Recently, the novel quadrupole time-of-flight mass spectrometry with $\mathrm{MS}^{\mathrm{E}}$ technique has been proven to be a powerful and reliable analytical approach for metabolite identification [16-20]. UPLC Q-TOF/HDMS with $\mathrm{MS}^{\mathrm{E}}$ technique is becoming increasingly popular in the analysis of biological fluids in the field of metabolomics because it provides high resolution, accurate mass measurement, and structural information [14, 16-20]. A method using UPLC QTOF/HDMS/MS ${ }^{\mathrm{E}}$-based metabolomics combined with multivariate statistical analysis was applied to rapidly identify urinary metabolite profiling of diet-induced hyperlipidaemia in a rat model. Orthogonal partial least squares discriminant analysis (OPLS-DA), correlation analysis, heat map analysis, and KEGG pathways analysis were performed for investigating the metabolic changes of diet-induced hyperlipidaemia and control rats, and the potential biomarkers were identified accordingly.

\section{Materials and Methods}

2.1. Animals and Sample Collection. The study was conducted in accordance with the Regulations of Experimental Animal Administration issued by the State Committee of Science and Technology of People's Republic of China. All procedures and the care of the rats were in accordance with institutional guidelines for animal use in research. Male Sprague-Dawley rats were obtained from the Central Animal Breeding House of Fourth Military Medical University (Xi'an, China). They were maintained at a constant humidity (ca. 60\%) and temperature $\left(\mathrm{ca} .23^{\circ} \mathrm{C}\right.$ ) with a light/dark cycle of $12 \mathrm{~h}$. Male rats underwent an adaptation period of several days, during which they were fed a commercial feed. After that they were separated randomly into two groups ( $n=8$ /group). Rats were randomly assigned into a diet-induced hyperlipidaemia group and control group. The control group was fed with the common diet during the whole experimental period, and the diet-induced hyperlipidaemia group was fed with high fat diets including $81 \%$ basic diet, $10 \%$ yolk powder, $7.5 \%$ lards, $0.3 \%$ sodium cholate, $0.2 \%$ methylthiouracil, and $1 \%$ cholesterol for continuous 6 weeks. After 6 weeks, individual rats were placed in metabolic cages (1 per cage) to obtain 24 hour urine collections. When urine samples were collected, rats were only freely accessible to water. All the samples were stored at $-80^{\circ} \mathrm{C}$ before analysis.

2.2. Sample Preparation. Prior to analysis, urine samples were thawed at room temperature and then centrifuged at $13000 \mathrm{rpm}$ for $10 \mathrm{~min}$ to remove solid materials. The supernatant was diluted at a ratio of $3: 1$ with distilled water, mixed, and centrifuged for UPLC analysis.

2.3. Chromatographic Separation. The UPLC analysis was performed on a Waters ACQUITY Ultra Performance LC system (Waters, USA) equipped with a Waters Xevo G2 QTof MS. Chromatographic separation was carried out at $45^{\circ} \mathrm{C}$ on an ACQUITY UPLC HSS T3 column $(2.1 \mathrm{~mm}$ $\times 100 \mathrm{~mm}, 1.8 \mu \mathrm{m})$. The mobile phase consisted of water (A) and acetonitrile (B), each containing $0.1 \%$ formic acid. The optimized UPLC elution conditions were $0-0.5 \mathrm{~min}, 1 \%$ B; $0.5-12.0 \mathrm{~min}, 1-30 \% \mathrm{~B}$; $12.0-15.0 \mathrm{~min}, 30-99 \% \mathrm{~B} ; 15.0-$ $16.0 \mathrm{~min}, 99 \% \mathrm{~B} ; 16.0-20.0 \mathrm{~min}, 99.0-1.0 \% \mathrm{~B}$. The flow rate 
was $0.45 \mathrm{~mL} / \mathrm{min}$. The autosampler was maintained at $4^{\circ} \mathrm{C}$. Every $2 \mu \mathrm{L}$ sample solution was injected for each run.

\subsection{Mass Spectrometry. Mass spectrometry was performed} on a quadrupole and orthogonal acceleration time-of-flight tandem mass spectrometer. The scan range was from 50 to $1200 \mathrm{~m} / z$. For both positive and negative electrospray modes, the capillary and cone voltage were set at $2.5 \mathrm{kV}$ and $45 \mathrm{~V}$, respectively. The desolvation gas was set at $900 \mathrm{~L} / \mathrm{h}$ at a temperature of $550^{\circ} \mathrm{C}$; the cone gas was set at $50 \mathrm{~L} / \mathrm{h}$; the source temperature was set at $120^{\circ} \mathrm{C}$. The mass spectrometry was operated in $\mathrm{W}$ optics mode with 12,000 resolution using dynamic range extension. The data acquisition rate was set to $0.1 \mathrm{~s}$, with a $0.1 \mathrm{~s}$ interscan delay. All analyses were acquired using the LockSpray to ensure accuracy and reproducibility. Leucine-enkephalin was used as the lockmass at a concentration of $300 \mathrm{ng} / \mathrm{mL}$ and flow rate of $5 \mu \mathrm{L} / \mathrm{min}$. Data were collected in centroid mode, the LockSpray frequency was set at $10 \mathrm{~s}$, and data were averaged over 10 scans. All the acquisition and analysis of data were operated by Waters MassLynx v4.1 software.

2.5. Analytical Method Assessment. The precision and repeatability of this experiment were tested for assessment of the developed UPLC-MS method according to different chemical polarities and $\mathrm{m} / z$ values; 8 ions including $\mathrm{m} / z$ 284.2934, 340.1060 , 282.2779, 256.2620, 367.1490, 296.2360, 372.2366, and 330.0618 were extracted for the assessment according to the variation of their peak areas and retention times. The six parallel random samples were injected to evaluate the sample preparation repeatability. Sample of quality control (QC) was injected. There were six control rats and six diet-induced hyperlipidemia rats; six batches of data from one QC sample could be obtained to evaluate the stability of the UPLC-MS system for the large-scale sample analysis.

2.6. Data Analysis. The raw data were analyzed using the MarkerLynx XS software. This software allowed deconvolution, alignment, and data reduction to give a list of mass and retention time pairs with corresponding intensities for all the detected peaks from each data file in the data set. The main parameters were set as follows: retention time range $1-18 \mathrm{~min}$, mass range $50-1000 \mathrm{amu}$, minimum intensity $1 \%$, mass tolerance 0.01 , retention time window 0.20 , mass window 0.05 , marker intensity threshold 500, and noise elimination level 6. All of the data were normalized to the summed total ion intensity per chromatogram, and the resultant data matrices were introduced to the EZinfo 2.0 software for OPLS-DA. Metabolite peaks were assigned by $\mathrm{MS}^{\mathrm{E}}$ analysis or interpreted with available biochemical databases, such as HMD, ChemSpider, and KEGG. Potential markers were extracted from S-plots. Correlation analysis and heat maps were analyzed by MetaboAnalyst software. The other statistical analysis was performed by SPSS 11.0. The significant differences between control group and diet-induced hyperlipidemia group were assessed by analysis of variance (ANOVA) followed by $t$-test for multiple comparisons. When comparing with the control group, $P$ values less than 0.05 were considered significant.

\section{Results and Discussion}

3.1. Sample Preparation and UPLC-MS Analysis. Urine is a complex sample containing various endogenous and exogenous acidic, basic, and neutral compounds with high polarity. Sample preparation by conventional methods including solid-phase extraction or liquid-liquid extraction may lead to loss of high polarity and high hydrophilicity metabolites. While metabolomics is an untargeted analysis of the global changes in endogenous metabolites, conventional methods may cause loss of potential biomarker. Therefore, minimal sample preparation steps were performed on urinary samples in order to avoid the loss of the endogenous metabolites. Urinary samples were centrifuged and diluted prior to the direct injection into UPLC-MS.

The complexity of the urinary sample makes the separation very difficult and consequently results in severe ion suppression. UPLC employs sub- $2 \mu \mathrm{m}$ particle size column, which generates high efficiency to the compound separation and concurrently increases sensitivity and resolution. Thus, UPLC was applied to urinary metabolomics of diet-induced hyperlipidaemia rats in the positive and negative ESI modes. Representative base peak intensity (BPI) chromatogram of the urine of diet-induced hyperlipidaemia rats in the positive ESI mode is shown in Figure 1. Reproducibility was determined from six replicated analyses of the same urinary sample. The variations of $m / z$ values and retention times of selected peaks in positive ESI mode were less than $8 \mathrm{mDa}$ and $0.05 \mathrm{~min}$, respectively, and the relative standard deviations of peak area and retention time are below $2.9 \%$ and $0.78 \%$, respectively. These results indicated the excellent reproducibility and stability during the whole sequence.

3.2. Multivariate Data Analysis. Metabolomics aims at the comparison of samples from a control group and from a case group. The OPLS-DA is an extension of the partial least squares discriminant analysis which integrates an orthogonal signal correction filter to distinguish variations that are useful for prediction of a quantitative response from variations that are orthogonal to prediction. OPLS-DA was demonstrated as a powerful tool for the analysis of qualitative data structures. OPLS-DA score plot was performed on the urinary metabolites from diet-induced hyperlipidemia rats and control rats. According to UPLC-MS data, 8642 peaks of positive ions were detected and processed by MarkerLynx using the same acquiring method. Figure 2(a) shows the OPLS-DA score plot of the diet-induced hyperlipidemia rats and control rats. The OPLS-DA score plot revealed good fitness and high predictability of the OPLS-DA model with high statistical values of $R^{2}$ and $Q^{2}$. The $R^{2}$ and $Q^{2}$ values are 0.975 and 0.933 , respectively. S-plot is a tool for visualization and interpretation of multivariate classification models. It images both the covariance and correlation between the metabolites and the modeled class designation for identification of statistically significant and biochemically interesting 


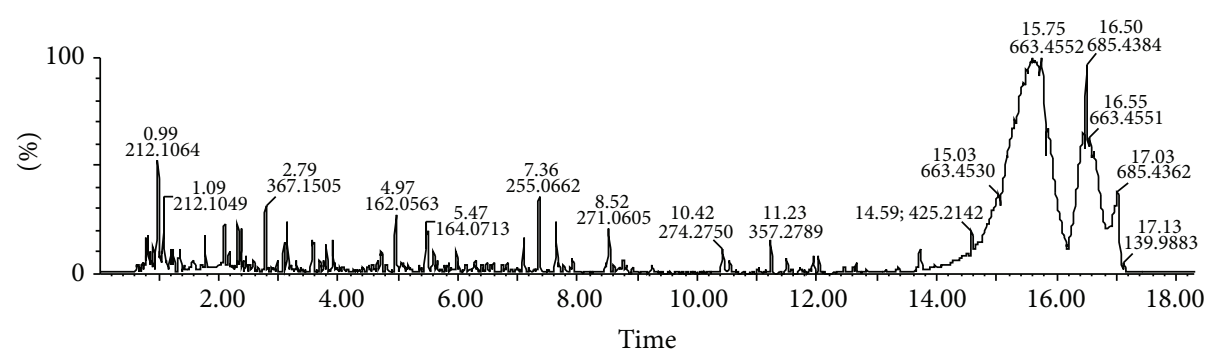

(a)

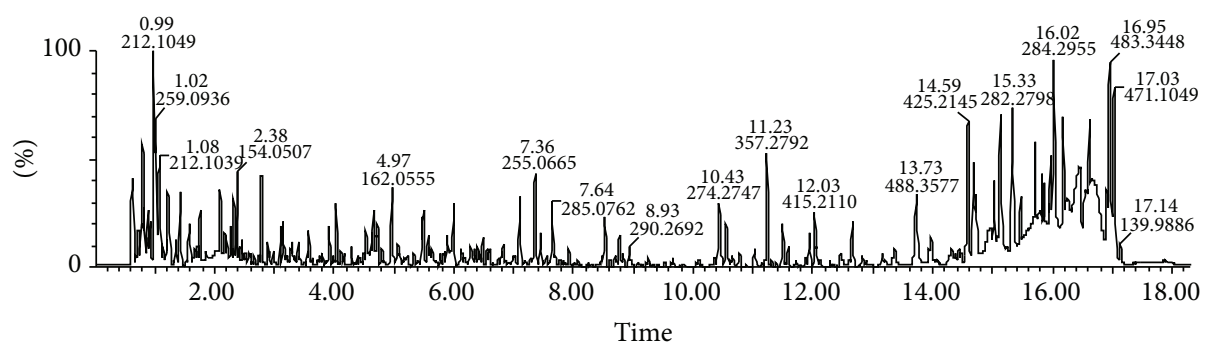

(b)

FIGURE 1: Base peak intensity (BPI) chromatograms obtained from the positive ion UPLC-MS analyses of control (a) and diet-induced hyperlipidemia (b) rats.

Scores comp[1] versus comp[2] colored by group (original)

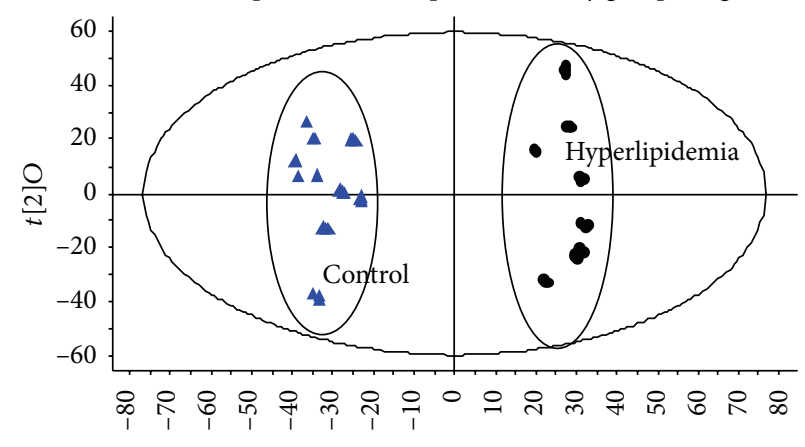

$t[1] P$

(a)

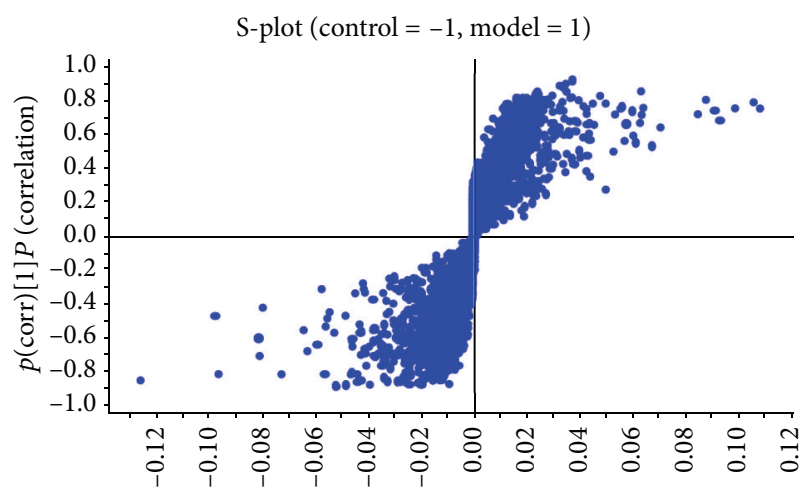

$p[1] P$ (loadings)

(b)

FIGURE 2: (a) OPLS-DA score plot based on the urinary metabolic profiling of the hyperlipidemia $(\bullet)$ and control ( $\mathbf{\Delta})$ rats; the results indicated that the urinary metabolic pattern was significantly changed in the diet-induced hyperlipidemia rats. (b) S-plot used in our biomarkers selection. The variables marked ( $\square$ ) are the metabolites selected as potential biomarkers. The significant metabolites were selected as potential biomarkers from S-plot and these urinary metabolites are associated with diet-induced hyperlipidemia.

metabolites which can avert the increase of false positives (type I error). The significant metabolites were selected as potential biomarkers from S-plot (Figure 2(b)). These urinary metabolites are associated with diet-induced hyperlipidemia.

\subsection{Identification of Potential Biomarkers. For metabolomics} research, the biggest challenge is the identification of potential biomarkers obtained from comparative samples, particularly when they are novel and published work on the compound class is unavailable or prior information is lacking otherwise. All the detected ions were arranged in descending order according to VIP (Variable Importance in the Projection) values, which reflect the influence of each metabolite in control rats and diet-induced hyperlipidemia rats. The more the variable deviates from the origin, the higher the VIP value will be obtained. According to the result of S-plot and reported methods, 16 variables were predicted by comparing the accurate $\mathrm{MS}$ and $\mathrm{MS}^{\mathrm{E}}$ fragments with the metabolites searching in ChemSpider, HMDB, and KEGG, and according to the possible fragment mechanisms, compounds without the given mass fragment information were removed from the candidate list and only the most probable compounds were 
TABLE 1: 13 biomarkers of hyperlipidemia detected by UPLC Q-TOF/MS in negative ion mode in the 4th week.

\begin{tabular}{|c|c|c|c|c|c|c|c|}
\hline Number & Mass & Metabolite & $\begin{array}{l}\text { i-FIT }{ }^{\mathrm{a}} \text {, elemental } \\
\text { composition }\end{array}$ & Molecular weight & Formula & Trend $^{\mathrm{b}}$ & Related pathway \\
\hline 1 & 284.2934 & Octadecanamide & $0.9, \mathrm{C}_{18} \mathrm{H}_{38} \mathrm{NO}$ & 283.4925 & $\mathrm{C}_{18} \mathrm{H}_{37} \mathrm{NO}$ & $\uparrow^{* * *}$ & Fatty acid metabolism \\
\hline 2 & 282.2779 & Oleamide & $1.2, \mathrm{C}_{18} \mathrm{H}_{36} \mathrm{NO}$ & 281.2718 & $\mathrm{C}_{18} \mathrm{H}_{35} \mathrm{NO}$ & $\uparrow^{* *}$ & Fatty acid metabolism \\
\hline 3 & 188.0713 & Tryptophan & $0.9, \mathrm{C}_{11} \mathrm{H}_{10} \mathrm{~N}_{1} \mathrm{O}_{2}$ & 204.2252 & $\mathrm{C}_{11} \mathrm{H}_{12} \mathrm{~N}_{2} \mathrm{O}_{2}$ & $\uparrow^{* *}$ & $\begin{array}{l}\text { Amino acid } \\
\text { metabolism }\end{array}$ \\
\hline 4 & 193.1235 & Citric acid & $0.7, \mathrm{C}_{6} \mathrm{H}_{9} \mathrm{O}_{7}$ & 192.1243 & $\mathrm{C}_{6} \mathrm{H}_{8} \mathrm{O}_{7}$ & $\downarrow^{* *}$ & TCA cycle \\
\hline 5 & 330.0618 & $\begin{array}{c}\text { Adenosine } 2^{\prime}, 3^{\prime} \text {-cyclic } \\
\text { phosphate }\end{array}$ & $1.0, \mathrm{C}_{10} \mathrm{H}_{13} \mathrm{~N}_{5} \mathrm{O}_{6} \mathrm{P}$ & 329.2059 & $\mathrm{C}_{10} \mathrm{H}_{12} \mathrm{~N}_{5} \mathrm{O}_{6} \mathrm{P}$ & $\downarrow^{* *}$ & Purine metabolism \\
\hline 6 & 393.3002 & Ursodeoxycholic acid & $0.8, \mathrm{C}_{24} \mathrm{H}_{41} \mathrm{O}_{4}$ & 392.5720 & $\mathrm{C}_{24} \mathrm{H}_{40} \mathrm{O}_{4}$ & $\uparrow^{* *}$ & Bile acid metabolism \\
\hline 7 & 114.0641 & Creatinine & 1.2, $\mathrm{C}_{4} \mathrm{H}_{8} \mathrm{~N}_{3} \mathrm{O}$ & 113.1179 & $\mathrm{C}_{4} \mathrm{H}_{7} \mathrm{~N}_{3} \mathrm{O}$ & $\uparrow^{* *}$ & Energy metabolism \\
\hline 8 & 281.0979 & Ascorbalamic acid & $0.9, \mathrm{C}_{9} \mathrm{H}_{17} \mathrm{~N}_{2} \mathrm{O}_{8}$ & 263.2014 & $\mathrm{C}_{9} \mathrm{H}_{13} \mathrm{NO}_{8}$ & $\uparrow^{* *}$ & $\begin{array}{l}\text { Carbohydrate } \\
\text { metabolism }\end{array}$ \\
\hline 9 & 259.0913 & 3-Methyluridine & 1.2, $\mathrm{C}_{10} \mathrm{H}_{15} \mathrm{~N}_{2} \mathrm{O}_{6}$ & 258.228 & $\mathrm{C}_{10} \mathrm{H}_{14} \mathrm{~N}_{2} \mathrm{O}_{6}$ & $\uparrow^{* *}$ & $\begin{array}{l}\text { Nucleoside } \\
\text { metabolism }\end{array}$ \\
\hline 10 & 212.1025 & 3-O-Methyldopa & 1.7, $\mathrm{C}_{10} \mathrm{H}_{14} \mathrm{NO}_{4}$ & 211.2145 & $\mathrm{C}_{10} \mathrm{H}_{13} \mathrm{NO}_{4}$ & $\downarrow^{* *}$ & $\begin{array}{l}\text { Amino acid } \\
\text { metabolism }\end{array}$ \\
\hline 11 & 162.1108 & Proline & 0.7, $\mathrm{C}_{7} \mathrm{H}_{13} \mathrm{O}_{3}$ & 115.1305 & $\mathrm{C}_{7} \mathrm{H}_{12} \mathrm{O}_{3}$ & $\downarrow^{* *}$ & $\begin{array}{l}\text { Amino acid } \\
\text { metabolism }\end{array}$ \\
\hline 12 & 282.1216 & 1-Methyladenosine & $1.2, \mathrm{C}_{11} \mathrm{H}_{16} \mathrm{~N}_{5} \mathrm{O}_{4}$ & 281.2679 & $\mathrm{C}_{11} \mathrm{H}_{15} \mathrm{~N}_{5} \mathrm{O}_{4}$ & $\downarrow^{* *}$ & $\begin{array}{l}\text { Nucleoside } \\
\text { metabolism }\end{array}$ \\
\hline 13 & 162.0538 & $\begin{array}{l}\text { Indole-3-carboxylic } \\
\text { acid }\end{array}$ & $0.8, \mathrm{C}_{9} \mathrm{H}_{8} \mathrm{NO}_{2}$ & 161.1574 & $\mathrm{C}_{9} \mathrm{H}_{7} \mathrm{NO}_{2}$ & $\uparrow^{* *}$ & $\begin{array}{l}\text { Amino acid } \\
\text { metabolism }\end{array}$ \\
\hline 14 & 368.1594 & Tryptophyl-tyrosine & $1.2, \mathrm{C}_{20} \mathrm{H}_{22} \mathrm{~N}_{3} \mathrm{O}_{4}$ & 367.3984 & $\mathrm{C}_{20} \mathrm{H}_{21} \mathrm{~N}_{3} \mathrm{O}_{4}$ & $\uparrow^{* *}$ & $\begin{array}{l}\text { Amino acid } \\
\text { metabolism }\end{array}$ \\
\hline 15 & 166.0708 & Phenylalanine & $0.9, \mathrm{C}_{9} \mathrm{H}_{12} \mathrm{NO}_{2}$ & 165.1891 & $\mathrm{C}_{9} \mathrm{H}_{11} \mathrm{NO}_{2}$ & $\downarrow^{* *}$ & $\begin{array}{l}\text { Amino acid } \\
\text { metabolism }\end{array}$ \\
\hline 16 & 126.0643 & 5-Methylcytosine & $1.3, \mathrm{C}_{5} \mathrm{H}_{8} \mathrm{~N}_{3} \mathrm{O}$ & 125.1286 & $\mathrm{C}_{5} \mathrm{H}_{7} \mathrm{~N}_{3} \mathrm{O}$ & $\downarrow^{* *}$ & $\begin{array}{l}\text { Nucleoside } \\
\text { metabolism }\end{array}$ \\
\hline
\end{tabular}

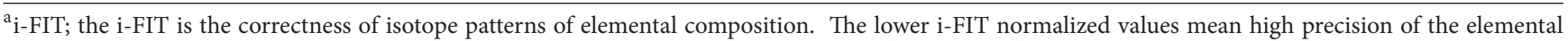
composition; ${ }^{b}$ change trend of hyperlipidemia rats versus control rats. The potential biomarkers were labeled with $(\downarrow)$ downregulated and $(\uparrow)$ upregulated. ${ }^{*} P<0.05$ and ${ }^{* *} P<0.01$.

reserved [21, 22]. MassLynx i-FIT algorithm is used to screen suggested elemental compositions by the likelihood that the isotopic pattern of the elemental composition matches a cluster of peaks in the spectrum, increasing confidence in identified compounds and simplifying results. The lower the i-FIT value, the better the fit [23]. By comparing the retention times and mass spectra to the authentic chemicals, 16 compounds were tentatively identified and shown in Table 1 .

\subsection{Study of Metabolic Changes and Biochemical Interpreta-} tion in Diet-Induced Hyperlipidemia Rats. Differences in the levels of metabolites between diet-induced hyperlipidemia rats and control rats were compared using OPLS-DA. The metabolic profiling and multivariate pattern recognition may observe a wider range of metabolites. Since metabolites can be regulated through a number of metabolic pathways, an investigation of the overall features rather than of several selected metabolites enabled us to understand the underlying pathophysiological status more comprehensively.

To investigate the change of identified metabolites in dietinduced hyperlipidemia rats, relative intensity of identified metabolites compared diet-induced hyperlipidemia rats with control rats. Increased urinary octadecanamide, oleamide, tryptophan, ursodeoxycholic acid, creatinine, ascorbalamic acid, 3-methyluridine, indole-3-carboxylic acid, and tryptophyl-tyrosine and decreased urinary citric acid, adenosine $2^{\prime}, 3^{\prime}$-cyclic phosphate, 3-O-methyldopa, proline, 1methyladenosine, phenylalanine, and 5-methylcytosine were observed in diet-induced hyperlipidemia rats (Figure 3(a)). Above-mentioned metabolites might play important roles in the metabolic changes of diet-induced hyperlipidemia rats. OPLS-DA loading plot was also generated in diet-induced hyperlipidemia rats and control rats. The loading plot indicated that identified metabolites were quantitatively higher or lower in diet-induced hyperlipidemia rats compared with control rats. Concentrations of identified metabolites including octadecanamide, oleamide, tryptophan, ursodeoxycholic acid, creatinine, ascorbalamic acid, 3-methyluridine, indole3-carboxylic acid, and tryptophyl-tyrosine were significantly increased in diet-induced hyperlipidemia rats, whereas the concentrations of citric acid, adenosine $2^{\prime}, 3^{\prime}$-cyclic phosphate, 3-O-methyldopa, proline, 1-methyladenosine, phenylalanine, and 5-methylcytosine were decreased in dietinduced hyperlipidemia rats (Figure 3(a)), which are in agreement with the changes of relative intensity (Figure $3(\mathrm{~b})$ ). The 


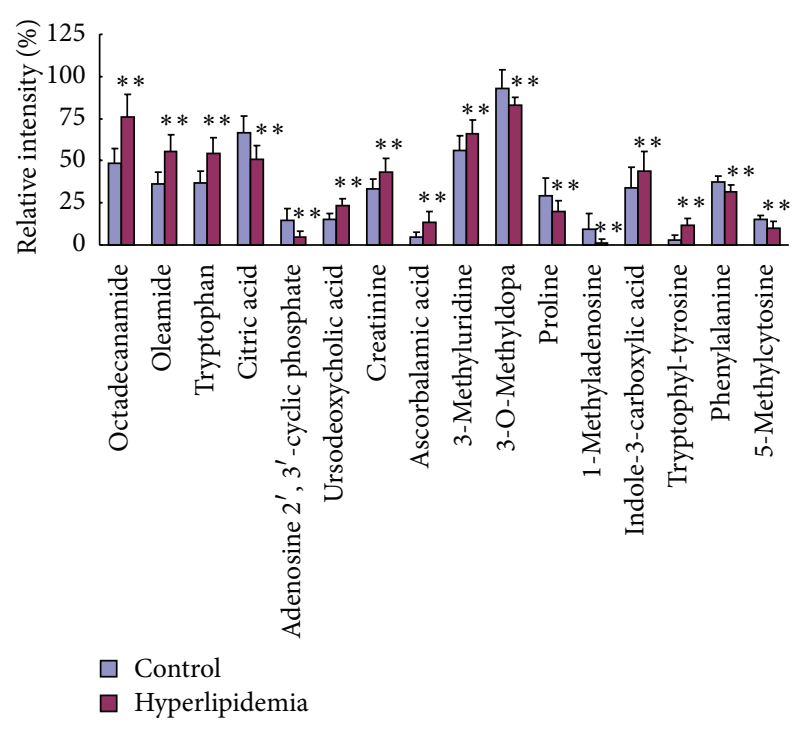

(a)

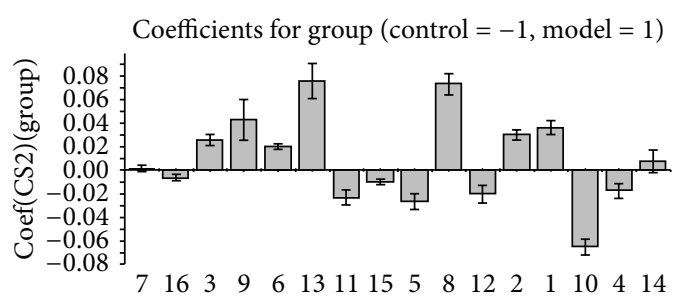

(b)

FIgURE 3: (a) Comparison of the relative intensity and (b) OPLSDA loading plot of putative potential biomarkers in control and diet-induced hyperlipidaemia rats. The loading plots represent which metabolites are quantitatively higher or lower in diet-induced hyperlipidaemia rats compared with control rats. Numbers consist with Table 1 .

results demonstrated that these upregulated or downregulated metabolites are associated with diet-induced hyperlipidemia.

To further understand the metabolic differences between diet-induced hyperlipidemia rats and control rats, identified metabolites were analyzed using correlation analysis and clustering heat maps. Correlation analysis showed the relation of differential metabolites. From the above plots, identified metabolites could be considered as being responsible for the separation between diet-induced hyperlipidemia rats and control rats and were therefore regarded as potential biomarkers. Heat map showed directly the variation of each metabolite, and the identified metabolites were visualized in a clustering heat map. Identified metabolites are showed in heat map, which shows the relative increase (red) or decrease (green) compared with control rats (Figure 4(a)). The hyperlipidemia model was capable of distinguishing diet-induced hyperlipidemia rats from control rats. The metabolites octadecanamide, oleamide, tryptophan, ursodeoxycholic acid, creatinine, 3-methyluridine, indole-3-carboxylic acid, and tryptophyl-tyrosine showed an increasing tendency in diet-induced hyperlipidemia rats.
Although these metabolites showed similar tendencies to increase, some of the metabolites showed different increasing levels in diet-induced hyperlipidemia rats. In contrast, the metabolites citric acid, adenosine $2^{\prime}, 3^{\prime}$-cyclic phosphate, 3 O-methyldopa, proline, 1-methyladenosine, phenylalanine, and 5-methylcytosine showed a decreasing tendency in dietinduced hyperlipidemia rats. These results are consistent with the relative intensity and OPLS-DA loading plots.

Amino acids serve as substrates for protein synthesis, metabolic energy (oxidation through TCA cycle), or gluconeogenesis and ketogenesis [24]. Increased urinary tryptophan and tryptophyl-tyrosine and decreased urinary phenylalanine were observed in diet-induced hyperlipidemia rats. Tryptophan is essential amino acid which cannot be synthesized by the body. Tryptophan either participates in proteins or is broken down for energy and metabolic intermediates. Dopamine is an important neurotransmitter as 5-hydroxytryptamine, which is derived from tryptophan metabolism. 3-O-Methyldopa is one of the main biochemical markers for aromatic amino acid decarboxylase deficiency, which affects dopamine biosynthesis. An obvious decrease of 3-O-methyldopa was observed in diet-induced hyperlipidemia rats compared with control rats. Indole-3carboxylic acid is the metabolite of tryptophan, the precursor of neurotransmitter 5-hydroxytryptamine. Level of indole-3carboxylic acid was significantly increased in diet-induced hyperlipidemia rats compared with control rats. Phenylalanine is an essential amino acid and its hydroxylation by phenylalanine hydroxylase to tyrosine is the major metabolic pathway for phenylalanine. Significant fate of tyrosine is a conversion to the catecholamines, for example, dopamine, norepinephrine, and epinephrine [25]. Diet-induced hyperlipidemia associated with dysfunction of tryptophan and phenylalanine, which were identified as potential biomarkers for hyperlipidemia or atherosclerosis, has been demonstrated $[9,26]$. Proline is the catabolite of peptide degradation by proline iminopeptidase and is a precursor of pyruvate. Pyruvate can be converted into acetyl-CoA, which is the main input for a series of reactions known as the TCA cycle. Decreases in levels of proline and citric acid were observed in diet-induced hyperlipidemia rats. Decrease in proline was related to the glutamate/P5C synthase pathway via inactivating P5C synthase or other enzymes involved [27]. The previous study demonstrated that urinary citric acid was significantly decreased in diet-induced hyperlipidemia [9]. These results indicated that amino acids metabolism and TCA cycle were disturbed in diet-induced hyperlipidemia rats.

Increased 3-methyluridine and decreased 1-methyladenosine and 5-methylcytosine were observed in diet-induced hyperlipidemia rats. It has been demonstrated that 3methyluridine and 1-methyladenosine were significantly increased in the pathogenesis of Alzheimer's disease [28]. 1-Methyladenosine is one of the modified nucleosides; the level is elevated in urine of patients with malignant tumors. Examination of expression of 1-methyladenosine is expected to be useful for the histological diagnosis of intraocular tumors. However, the previous study did not report that 3-methyluridine, 1-methyladenosine, and 5-methylcytosine 


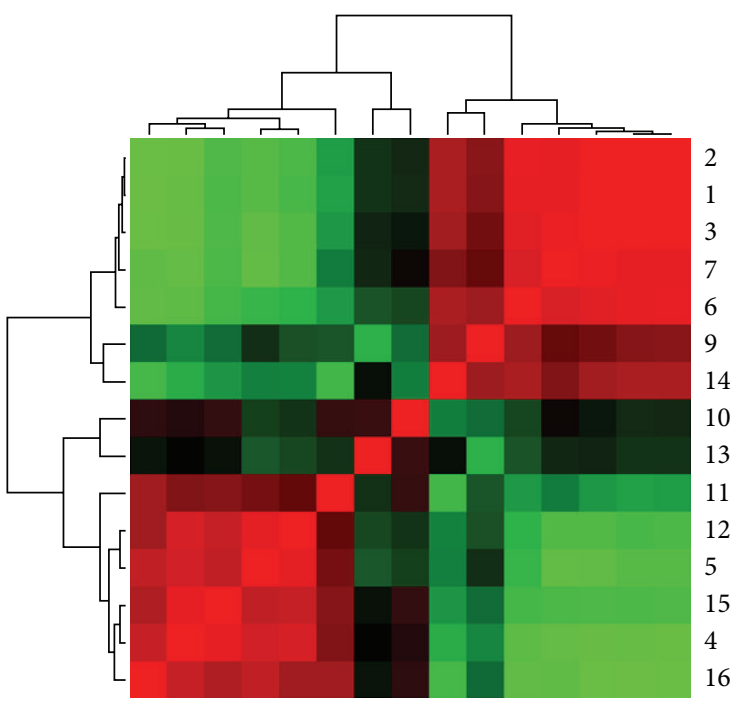

$\begin{array}{lllllllllllllll}16 & 4 & 15 & 5 & 12 & 11 & 13 & 10 & 14 & 9 & 6 & 7 & 3 & 1 & 2\end{array}$

Color key

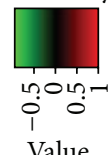

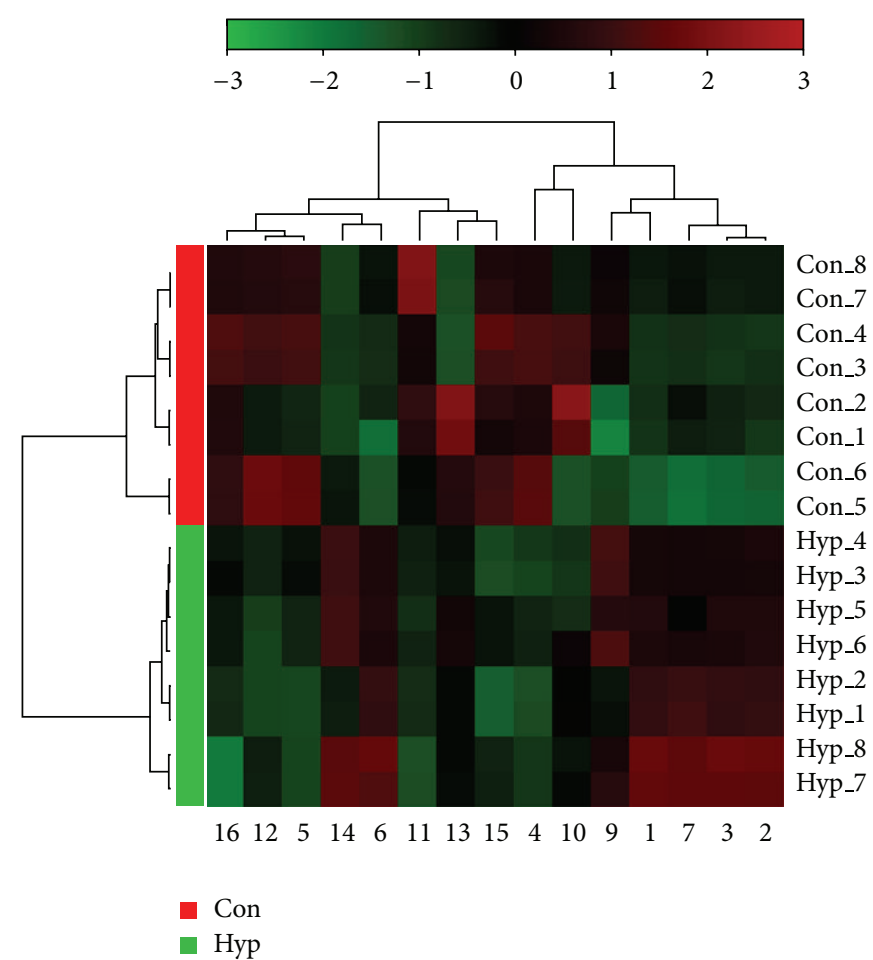

(b)

(a)

FIGURE 4: Correlation analysis (a) of the differential metabolites in control rats and diet-induced hyperlipidaemia rats. Heat map (b) for identified metabolites in control rats and diet-induced hyperlipidaemia rats. The color of each section is proportional to the significance of change of metabolites (red, upregulated; green, downregulated). Rows: samples; columns: metabolites. Numbers consist with Table 1.

were identified as potential biomarkers in diet-induced hyperlipidemia rats.

The concentrations of octadecanamide and oleamide were higher in diet-induced hyperlipidemia rats than in control rats. Fatty acids are reported to be associated with atherosclerotic and inflammatory diseases because they are the major components of the cytoplasmic membrane and the precursor fatty acids for prostaglandins and leukotrienes [29]. Bile acids have many important physiological functions such as lipid absorption, cholesterol homeostasis, and generation of bile flow that help in the recirculation and excretion of exogenous and endogenous metabolites. Increased urinary ursodeoxycholic acid was observed in dietinduced hyperlipidemia rats compared with control rats. It has been demonstrated that increased ursodeoxycholic acid was associated with atherosclerosis and ursodeoxycholic acid was identified as a potential biomarker in atherosclerosis rats [26]. In addition, the previous study demonstrated that urinary cholesterol was significantly increased in dietinduced hyperlipidemia [12]. Creatinine is usually produced at a relatively constant rate by the human body. Although serum creatinine is a commonly used indicator of renal function, increased urinary creatinine is observed only when significant injury occurs in renal function. Therefore, an increased level of urinary creatinine observed in the dietinduced hyperlipidaemia rats might indicate renal injury caused by hyperlipidaemia.

\section{Conclusions}

Urinary metabolomics based on UPLC Q-TOF/HDMS, a novel $\mathrm{MS}^{\mathrm{E}}$ data collection technique, and a multivariate statistical technique has been used to study diet-induced hyperlipidaemia in a rat model. The OPLS-DA score plot showed the complete distinction of diet-induced hyperlipidaemia rats and control rats. Furthermore, significant differences in the urinary levels of fatty acids, amino acids, nucleosides, and bile acids were observed in diet-induced hyperlipidaemia rats. These results demonstrated the perturbations of fatty acids metabolism, amino acid metabolism, and nucleosides metabolism of diet-induced hyperlipidaemia. This research also demonstrated that UPLC-MS-based metabolomics was a promising tool to find and identify potential biomarkers in diet-induced hyperlipidaemia rats.

\section{Conflict of Interests}

There is no conflict of interests to declare.

\section{Authors' Contribution}

Hua Miao and Hua Chen contributed to this paper as co-first authors. 


\section{Acknowledgments}

This study was supported by the Program for the New Century Excellent Talents in University (NCET-13-0954) and the Changjiang Scholars and Innovative Research Team in University (IRT1174) from Ministry of Education of China, the National Natural Science Foundation of China (J1210063, 81001622, and 81073029), National Innovation Training Plan Program (201310697004), the Project “As a Major New Drug to Create a Major National Science and Technology Special" (2011ZX09401-308-034, 2014ZX09304-307-02), and the China Postdoctoral Science Foundation (2012M521831).

\section{References}

[1] J. K. Nicholson, J. C. Lindon, and E. Holmes, "Metabonomics': understanding the metabolic responses of living systems to pathophysiological stimuli via multivariate statistical analysis of biological NMR spectroscopic data," Xenobiotica, vol. 29, no. 11, pp. 1181-1189, 1999.

[2] J. K. Nicholson, J. Connelly, J. C. Lindon, and E. Holmes, "Metabonomics: a platform for studying drug toxicity and gene function," Nature Reviews Drug Discovery, vol. 1, no. 2, pp. 153$161,2002$.

[3] J. C. Lindon, E. Holmes, M. E. Bollard, E. G. Stanley, and J. K. Nicholson, "Metabonomics technologies and their applications in physiological monitoring, drug safety assessment and disease diagnosis," Biomarkers, vol. 9, no. 1, pp. 1-31, 2004.

[4] J. H. O'Keefe and D. S. H. Bell, "Postprandial hyperglycemia/hyperlipidemia (postprandial dysmetabolism) is a cardiovascular risk factor," The American Journal of Cardiology, vol. 100, no. 5, pp. 899-904, 2007.

[5] E. Esteve, W. Ricart, and J. M. Fernández-Real, "Dyslipidemia and inflammation: an evolutionary conserved mechanism," Clinical Nutrition, vol. 24, no. 1, pp. 16-31, 2005.

[6] J. Genest, R. McPherson, J. Frohlich et al., "2009 Canadian Cardiovascular Society/Canadian guidelines for the diagnosis and treatment of dyslipidemia and prevention of cardiovascular disease in the adult-2009 recommendations," The Canadian Journal of Cardiology, vol. 25, no. 10, pp. 567-579, 2009.

[7] M. Adiels, S.-O. Olofsson, M.-R. Taskinen, and J. Borén, "Overproduction of very low-density lipoproteins is the hallmark of the dyslipidemia in the metabolic syndrome," Arteriosclerosis, Thrombosis, and Vascular Biology, vol. 28, no. 7, pp. 1225-1236, 2008.

[8] D. J. Wu, B. J. Zhu, and X. D. Wang, "Metabonomics-based omics study and atherosclerosis," Journal of Clinical Bioinformatics, vol. 1, no. 30, 2011.

[9] F. Liu, P. P. Gan, H. Wu, W. S. Woo, E. S. Ong, and S. F. Y. Li, “A combination of metabolomics and metallomics studies of urine and serum from hypercholesterolaemic rats after berberine injection," Analytical and Bioanalytical Chemistry, vol. 403, no. 3, pp. 847-856, 2012.

[10] X. Zhang, C. Wu, H. Wu et al., "Anti-hyperlipidemic effects and potential mechanisms of action of the caffeoylquinic acid-rich Pandanus tectorius fruit extract in hamsters fed a high fat-diet," PLoS ONE, vol. 8, no. 4, Article ID e61922, 2013.

[11] Q. Zhang, G.-J. Wang, J.-Y. A et al., "Application of GC/MSbased metabonomic profiling in studying the lipid-regulating effects of Ginkgo biloba extract on diet-induced hyperlipidemia in rats," Acta Pharmacologica Sinica, vol. 30, no. 12, pp. 16741687, 2009.
[12] Q. Zhang, G. Wang, J. A et al., "Metabonomic profiling of dietinduced hyperlipidaemia in a rat model," Biomarkers, vol. 15, no. 3, pp. 205-216, 2010.

[13] J.-P. F. Morand, J. Macri, and K. Adeli, "Proteomic profiling of hepatic endoplasmic reticulum-associated proteins in an animal model of insulin resistance and metabolic dyslipidemia," The Journal of Biological Chemistry, vol. 280, no. 18, pp. 1762617633, 2005.

[14] Y. Y. Zhao, "Metabolomics in chronic kidney disease," Clinical Chimica Acta, vol. 422, pp. 59-69, 2013.

[15] M. Wrona, T. Mauriala, K. P. Bateman, R. J. Mortishire-Smith, and D. O'Connor, "All-in-One' analysis for metabolite identification using liquid chromatography/hybrid quadrupole timeof-flight mass spectrometry with collision energy switching," Rapid Communications in Mass Spectrometry, vol. 19, no. 18, pp. 2597-2602, 2005.

[16] Y.-Y. Zhao, J. Liu, X.-L. Cheng, X. Bai, and R.-C. Lin, "Urinary metabonomics study on biochemical changes in an experimental model of chronic renal failure by adenine based on UPLC Q-TOF/MS," Clinica Chimica Acta, vol. 413, no. 5-6, pp. 642649, 2012.

[17] Y. Y. Zhao, X. Shen, X. L. Cheng, F. Wei, X. Bai, and R. C. Lin, "Urinary metabonomics study on the protective effects of ergosta-4, 6, 8(14), 22-tetraen-3-one on chronic renal failure in rats using UPLC Q-TOF/MS and a novel $\mathrm{MS}^{\mathrm{E}}$ data collection technique," Process Biochemistry, vol. 47, no. 12, pp. 1980-1987, 2012.

[18] Y. Y. Zhao, Y. L. Feng, X. Bai, X. J. Tan, R. C. Lin, and Q. Mei, "Ultra performance liquid chromatography-based metabonomic study of therapeutic effect of the surface layer of Poria cocos on adenine-induced chronic kidney disease provides new insight into anti-fibrosis mechanism," PLoS ONE, vol. 8, no. 3, Article ID e59617, 2013.

[19] Y. Y. Zhao, P. Lei, D. Q. Chen, Y. L. Feng, and X. Bai, "Renal metabolic profiling of early renal injury and renoprotective effects of Poria cocos epidermis using UPLC QTOF/HSMS/MS ${ }^{\mathrm{E}}$," Journal of Pharmaceutical and Biomedical Analysis, vol. 81-82, pp. 202-209, 2013.

[20] Y. Y. Zhao, H. T. Li, Y. L. Feng, X. Bai, and R. C. Lin, "Urinary metabonomic study of the surface layer of Poria cocos as an effective treatment for chronic renal injury in rats," Journal of Ethnopharmacology, vol. 148, no. 2, pp. 403-410, 2013.

[21] Y. Y. Zhao, L. Zhang, F. Y. Long et al., "UPLC-QTOF/HSMS/MS ${ }^{\mathrm{E}}$-based metabonomics for adenine-induced changes in metabolic profiles of rat faeces and intervention effects of ergosta-4, 6, 8(14), 22-tetraen-3-one," ChemicoBiological Interactions, vol. 201, no. 1-3, pp. 31-38, 2013.

[22] Y.-Y. Zhao, X.-L. Cheng, F. Wei et al., "Intrarenal metabolomic investigation of chronic kidney disease and its TGF- $\beta 1$ mechanism in induced-adenine rats using UPLC QTOF/HSMS/MS ${ }^{\mathrm{E}}$," Journal of Proteome Research, vol. 12, no. 2, pp. 692-703, 2013.

[23] Y.-Y. Zhao, X.-L. Cheng, F. Wei, X. Bai, and R.-C. Lin, "Application of faecal metabonomics on an experimental model of tubulointerstitial fibrosis by ultra performance liquid chromatography/high-sensitivity mass spectrometry with $\mathrm{MS}^{\mathrm{E}}$ data collection technique," Biomarkers, vol. 17, no. 8, pp. 721-729, 2012.

[24] B. K. Lopansri, N. M. Anstey, G. J. Stoddard et al., "Elevated plasma phenylalanine in severe malaria and implications for pathophysiology of neurological complications," Infection and Immunity, vol. 74, no. 6, pp. 3355-3359, 2006. 
[25] J. D. Fernstrom and M. H. Fernstrom, "Tyrosine, phenylalanine, and catecholamine synthesis and function in the brain," The Journal of Nutrition, vol. 137, no. 6, pp. 1539S-1547S, 2007.

[26] F. Zhang, Z. Jia, P. Gao et al., "Metabonomics study of atherosclerosis rats by ultra fast liquid chromatography coupled with ion trap-time of flight mass spectrometry", Talanta, vol. 79, no. 3, pp. 836-844, 2009.

[27] M. Watford, "Glutamine metabolism and function in relation to proline synthesis and the safety of glutamine and proline supplementation," The Journal of Nutrition, vol. 138, no. 10, pp. 2003S-2007S, 2008.

[28] S. H. Lee, I. Kim, and B. C. Chung, "Increased urinary level of oxidized nucleosides in patients with mild-to-moderate Alzheimer's disease," Clinical Biochemistry, vol. 40, no. 13-14, pp. 936-938, 2007.

[29] J. Dyerberg, "Linolenate-derived polyunsaturated fatty acids and prevention of atherosclerosis," Nutrition reviews, vol. 44, no. 4, pp. 125-134, 1986. 

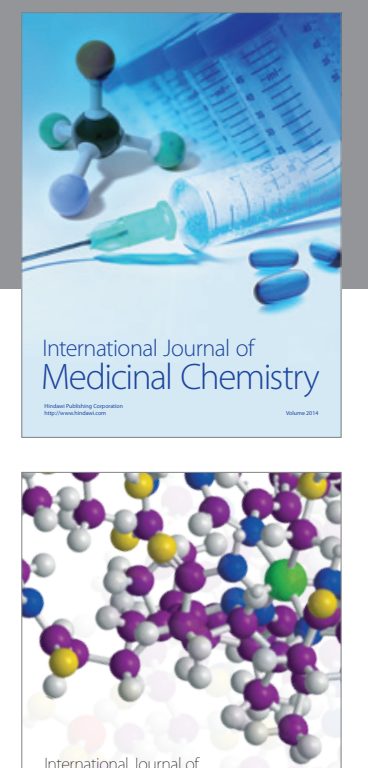

\section{Carbohydrate} Chemistry

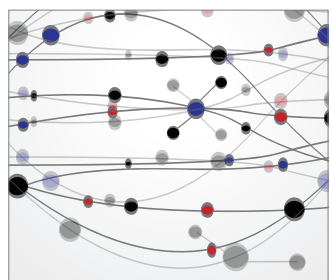

The Scientific World Journal
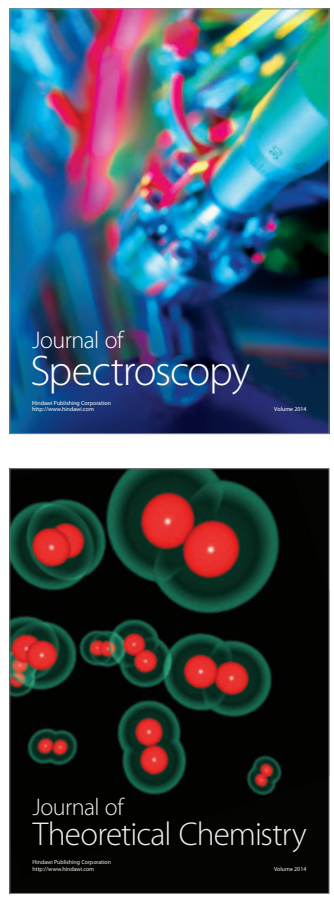
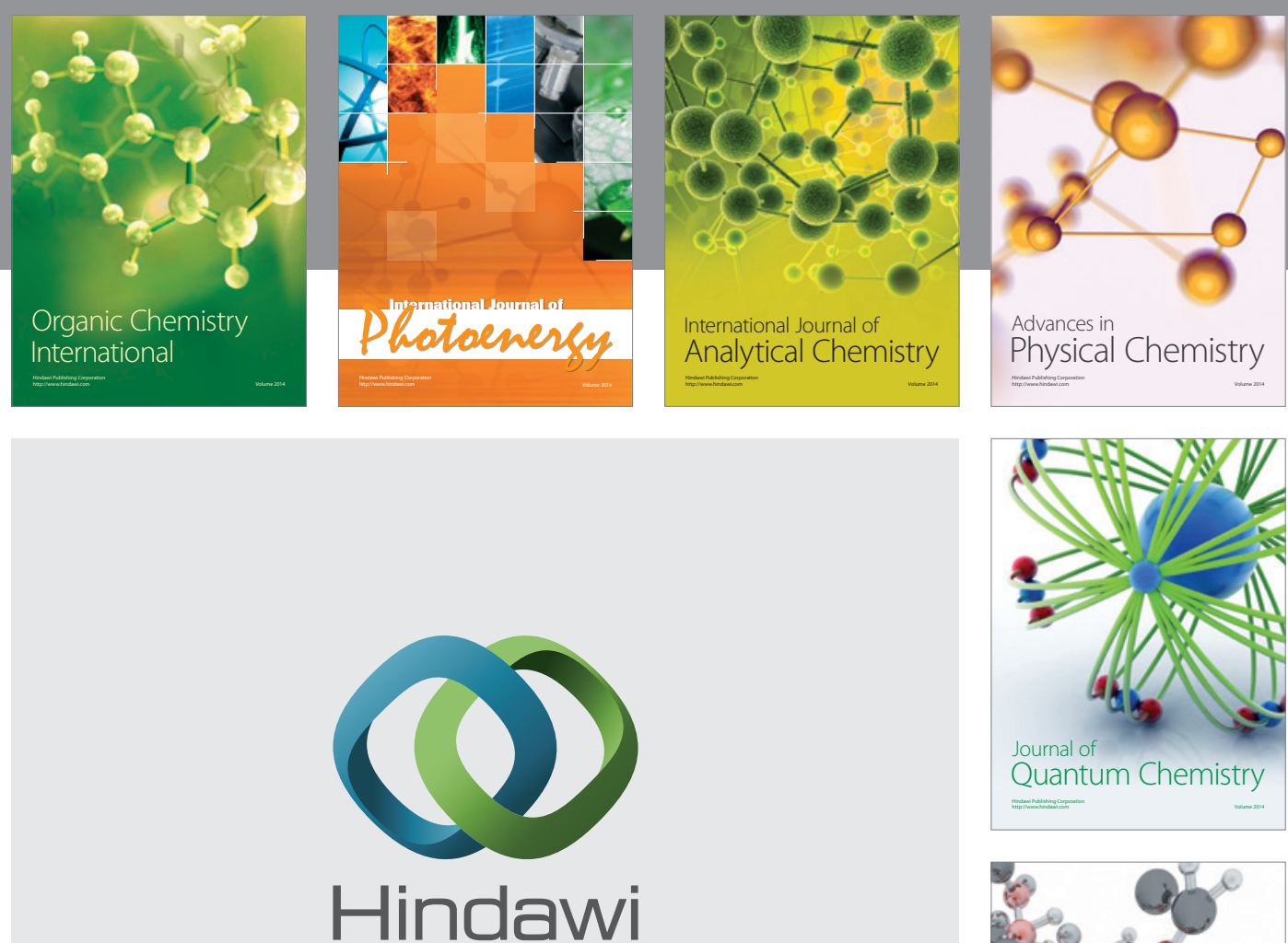

Submit your manuscripts at

http://www.hindawi.com

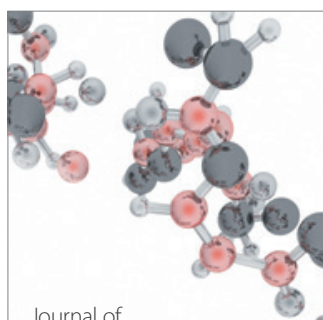

Analytical Methods

in Chemistry

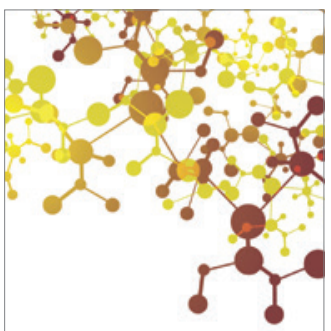

Journal of

Applied Chemistry

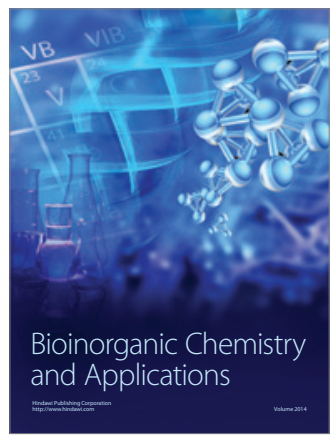

Inorganic Chemistry
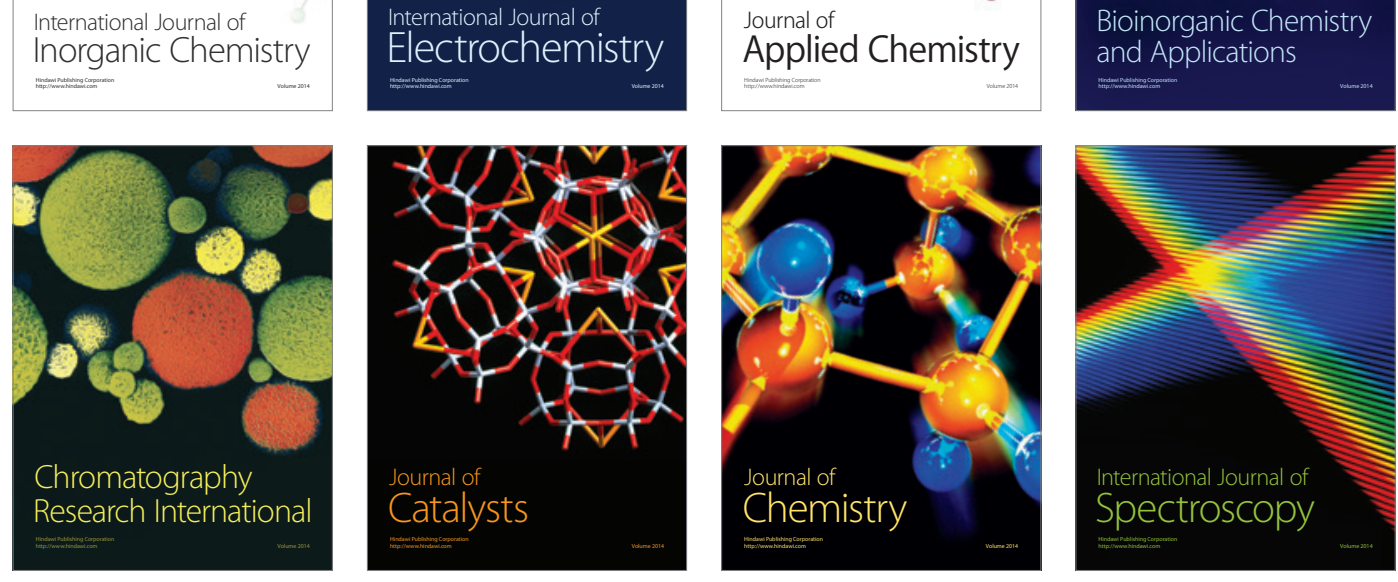